\title{
Modeling and analysis of biodegradation of xenobiotic polymers based on experimental results
}

\author{
M. Watanabe ${ }^{1} \quad$ F. Kawai ${ }^{2}$
}

(Received 31 July 2007; revised 11 February 2008)

\begin{abstract}
An endogenous depolymerization model based on uniform weight distribution is introduced. The time dependent model with temporal dependent degradation rate is reduced to a time independent model. The previously developed techniques were applied to an inverse problem to determine the degradation rate. The transition of weight distribution was simulated by solving an initial value problem. Those techniques are applied to degradation processes of polylactic acid. They are applicable to other polymers subject to endogenous depolymerization processes.
\end{abstract}

See http://anziamj.austms.org.au/ojs/index.php/ANZIAMJ/article/view/361 for this article, (C) Austral. Mathematical Soc. 2008. Published March 23, 2008. ISSN $1446-8735$ 


\section{Contents}

1 Introduction

C458

2 Derivation of model

C459

3 Determination of temporal factor

C462

4 Discussion

C468

References

C470

\section{Introduction}

Biodegradation is an essential factor for environmental protection against inadequate accumulation of xenobiotic polymers in the environment. Microbial depolymerization processes fall into one of two categories: exogenous type or endogenous type [1]. In exogenous depolymerization processes, the degradation is restricted only to the terminals of molecules, where monomer units are separated stepwise. Examples of polymers subject to exogenous depolymerization include polyethylene (PE) and polyethylene glycol (PEG). We proposed a mathematical model for PE biodegradation and introduced experimental results into analysis based on the model to determine degradation rates and to simulate transitions of weight distribution $[2,3,4,13,14,15]$. We adapted the exogenous depolymerization model developed for PE biodegradation to analysis of PEG depolymerization [16, 19].

Unlike exogenous depolymerization processes, molecules can split internally in endogenous depolymerization processes. Examples of endogenous depolymerization processes include the enzymatic degradation of polyvinyl alcohol (PVA). We proposed a mathematical model for enzymatic degradation of PVA, and introduced gel permeation chromatography (GPC) data 
before and after degradation of PVA [7] into analysis to determine degradation rates. We solved those problems numerically, and simulated the transition of the weight distribution $[12,13,17]$. The experimental and analytical study of endogenous depolymerization has continued to cover degradation of polylactic acid (PLA) [18]. Figure 1 shows the weight distribution of PLA before and after enzymatic degradation for five hours and sixty-seven hours. We formulated an inverse problem to determine the degradation rate for which the solution of the initial value problem also satisfies the weight distribution after five hours of incubation.

During incubation, PLA was dissolved in chloroform. As the time elapsed, chloroform was lost by evaporation, resulting in reduced degradation rates. In a previous study we considered the temporal change of degradability, and incorporated a temporally dependent degradation rate into the endogenous depolymerization model. We continue the study of endogenous depolymerization in this article. We introduce a mathematical model of endogenous depolymerization. We describe numerical techniques to analyze the model, and present numerical results.

\section{Derivation of model}

In order to formulate an enzymatic degradation process of a polymeric compound mathematically, let $w(t, M)$ be its weight distribution with respect to the molecular weight $M$ at time $t$. For $K \leq M$, let $p(t, K, M)$ denote the weight transition from $w(t, M)$ to $w(t, K)$ per unit time. We have shown $[12,13,17,18]$ that the functions $w(t, M)$ and $p(t, K, M)$ satisfy

$$
\frac{\partial w}{\partial t}(t, M)=-\int_{0}^{M} p(t, K, M) d K+\int_{M}^{\infty} p(t, M, K) d K .
$$

Let $\gamma(t, M)$ be the amount which $w(t, M)$ loses per unit time and unit weight. Then the rate of loss of $w(t, M)$ is $\gamma(t, M) w(t, M)$. Suppose that 


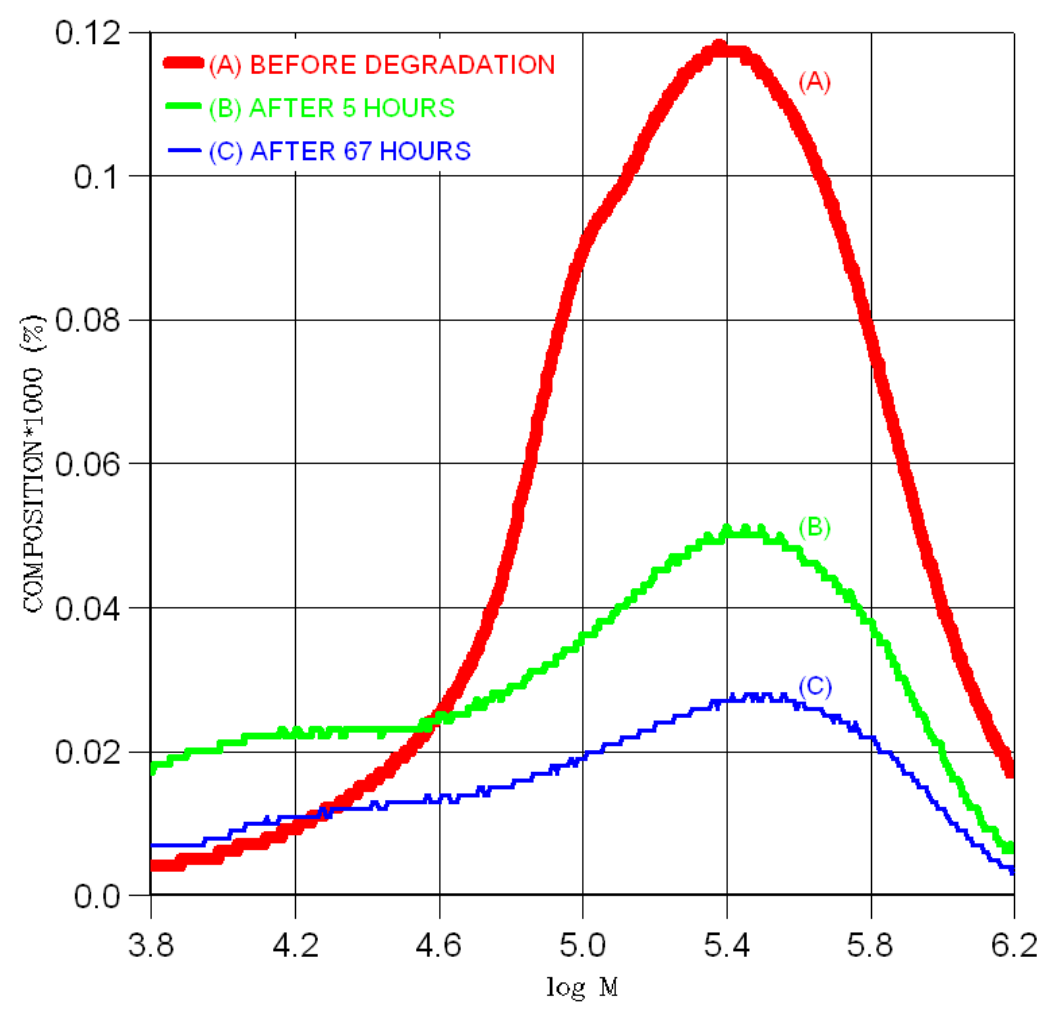

FiguRE 1: Weight distribution of PLA before and after enzymatic degradation. Residual amounts of PLA after 5 and $67 \mathrm{hr}$ incubation were $40 \%$ and $27 \%$, respectively [18]. 
this amount is uniformly distributed over the the interval $[0, M]$. Then $p(t, K, M)=(1 / M) \gamma(t, M) w(t, M)$, and we obtain, from equation (1),

$$
\frac{\partial w}{\partial t}(t, M)=-\gamma(t, M) w(t, M)+\int_{M}^{\infty} \frac{1}{K} \gamma(t, K) w(t, K) d K .
$$

When the initial weight distribution is given in terms of a prescribed function $f(M)$, the initial condition,

$$
w(0, M)=f(M),
$$

is imposed on the solution of equation (2). Given an additional condition

$$
w(T, M)=g(M),
$$

where $T>0$, the inverse problem requires the determination of the degradation rate $\gamma(t, K)$ for which the solution of the initial value problem (2)-(3) also satisfies the condition (4).

A time factor of degradability, such as concentration of enzyme or temperature, affects molecules regardless of their sizes, and we assume $\gamma(t, M)$ to be a product of a function of $t, \sigma(t)$, and a function of $M, \lambda(M)$ :

$$
\gamma(t, M)=\sigma(t) \lambda(M) .
$$

Let $\tau=\int_{0}^{t} \sigma(s) d s$, and $W(\tau, M)=w(t, M)$, which results in

$$
\frac{\partial W}{\partial \tau}=-\lambda(M) W+\int_{M}^{\infty} \frac{1}{K} \lambda(M) W(\tau, K) d K
$$

subject to the initial condition

$$
W(0, M)=f(M) .
$$

The inverse problem is to find the degradation rate $\lambda(M)$ for which the solution of the initial value problem (6)-(7) is also satisfied by the final condition

$$
W(\mathcal{T}, M)=g(M),
$$


where

$$
\mathcal{T}=\int_{0}^{T} \sigma(s) d s
$$

Watanabe and Kawai [17] developed numerical techniques to solve the inverse problem with a model proposed previously. We adopt these techniques for the current problem. We introduce the weight distribution before and after five hours of incubation shown in Figure 1 into the analysis to set the initial condition (7) and the final condition (8), and solved the inverse problem numerically for $\mathcal{T}=5 / 24$ [18]. Figure 2 shows the graph of the degradation rate $\lambda(M)$. Figure 3 shows the results of numerical simulations for the transition of weight distribution over ten hours of incubation based on the degradation rate shown in Figure 2. Figure 4 shows the comparison of the experimental result with a numerical result for the weight distribution after five hours of incubation. Figure 5 shows the comparison of the experimental result of weight distribution after sixty-seven hours of incubation with a numerical result for the weight distribution after incubation for 8.5 hours based on the degradation rate shown in Figure 2.

\section{Determination of temporal factor}

Since the decrease of degradability was due to evaporation of chloroform, we assume that $\sigma(t)$ is an exponential function of time:

$$
\sigma(t)=e^{-a t+b} .
$$

Then

$$
\tau=\int_{0}^{t} \sigma(s) d s=\int_{0}^{t} e^{-a s+b} d s=\frac{e^{b}}{a}\left(1-e^{-a t}\right) .
$$

Suppose that two sets of data $\left(T_{1}, \mathcal{T}_{1}\right)$ and $\left(T_{2}, \mathcal{T}_{2}\right)$ are available:

$$
\mathcal{T}_{1}=\int_{0}^{T_{1}} \sigma(s) d s
$$




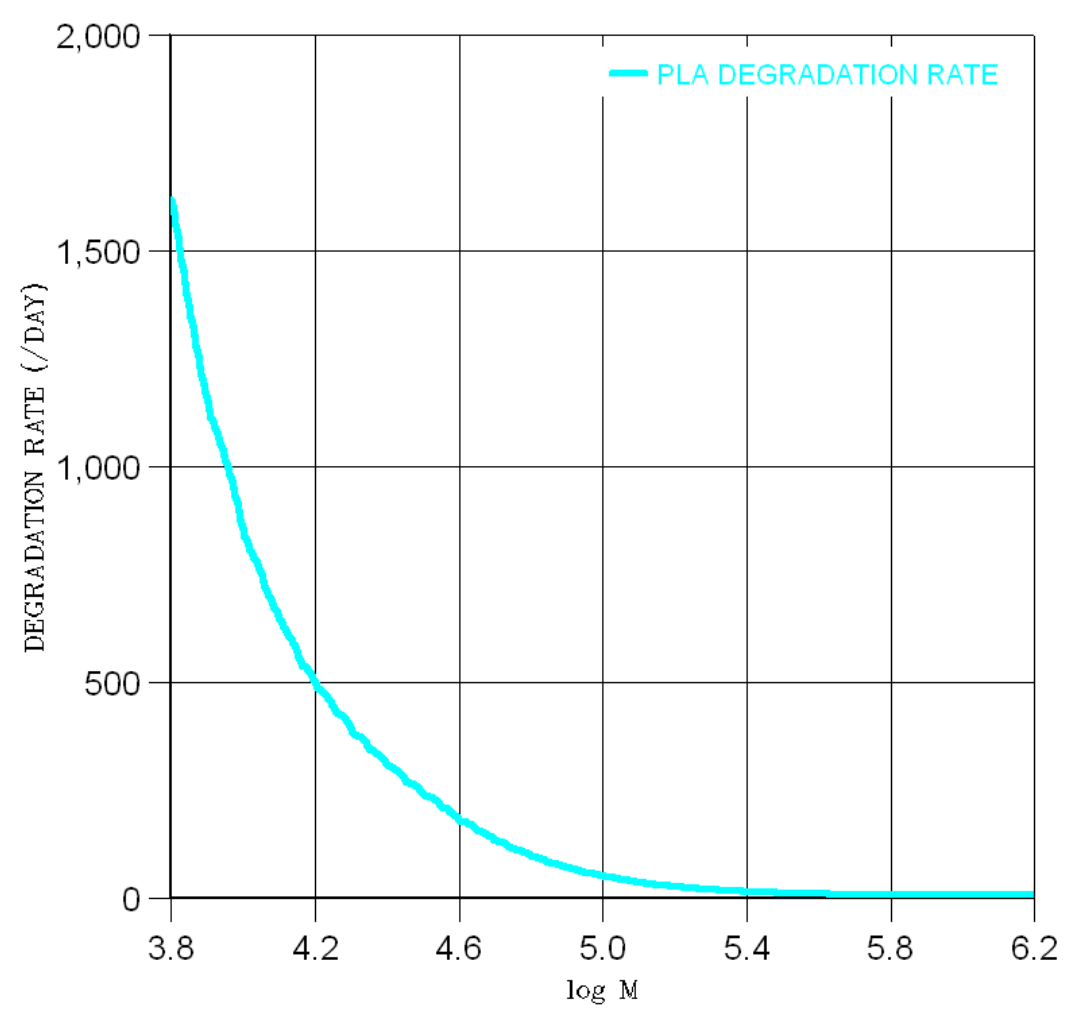

Figure 2: Degradation rate of PLA based on the GPC profiles obtained before and after incubation for five hours shown in the Figure 1. 


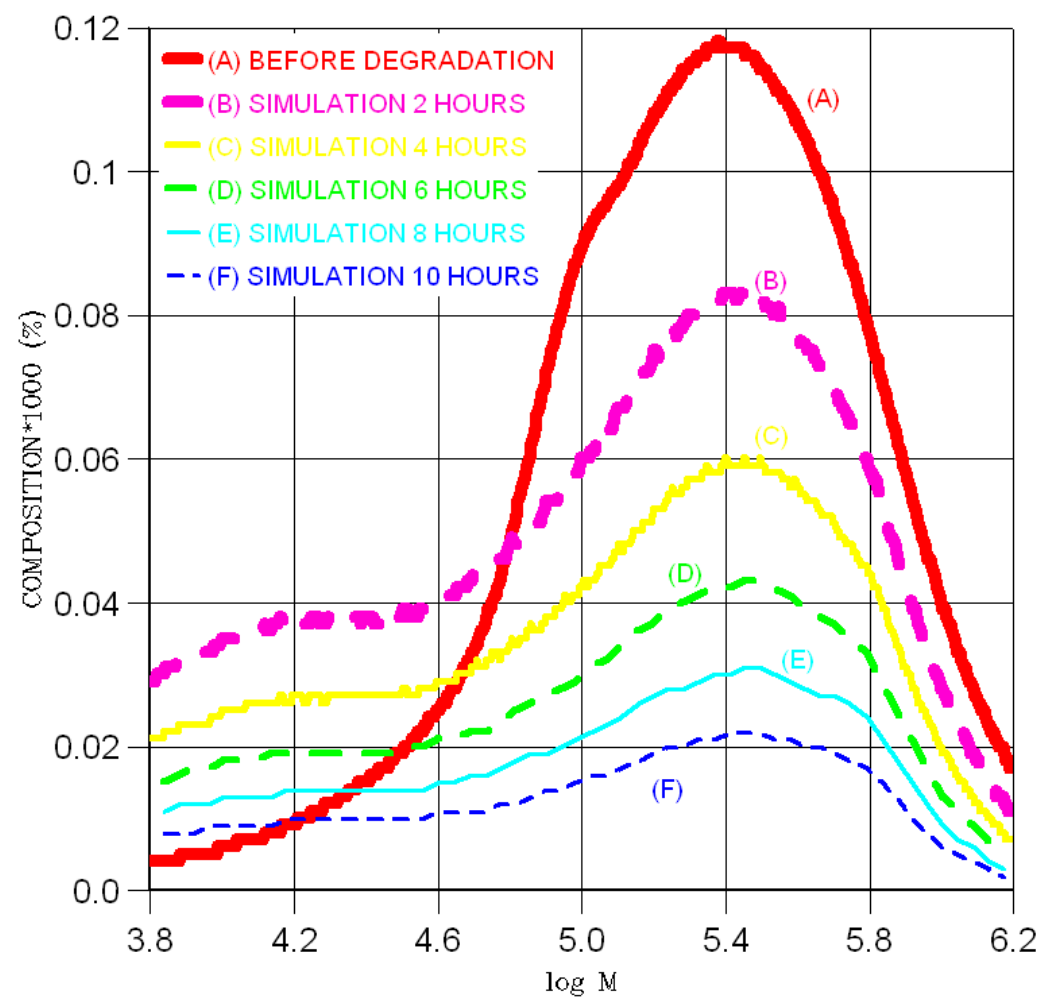

Figure 3: Transition of weight distribution for ten hours based on the degradation rate shown in the Figure 2. 


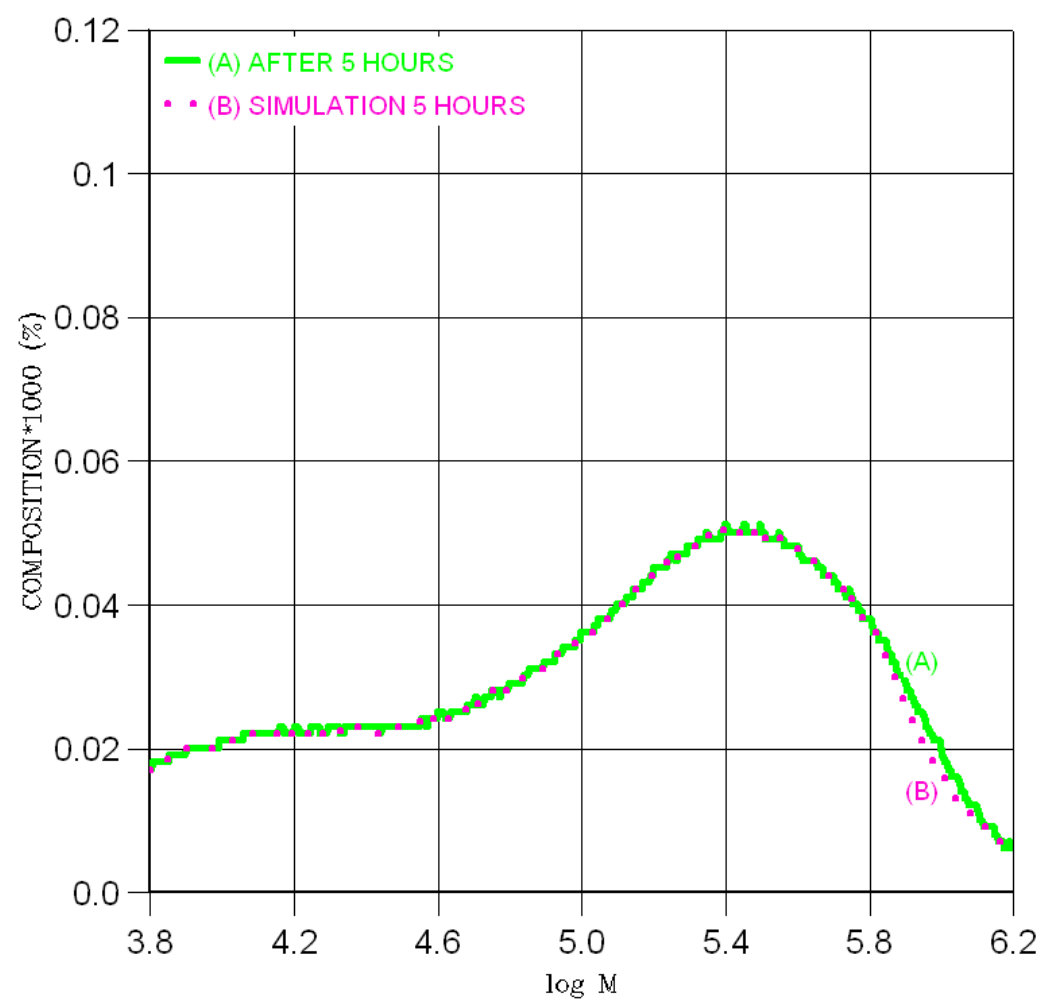

FigURE 4: Weight distribution after five hours of incubation and simulation for five hours. 


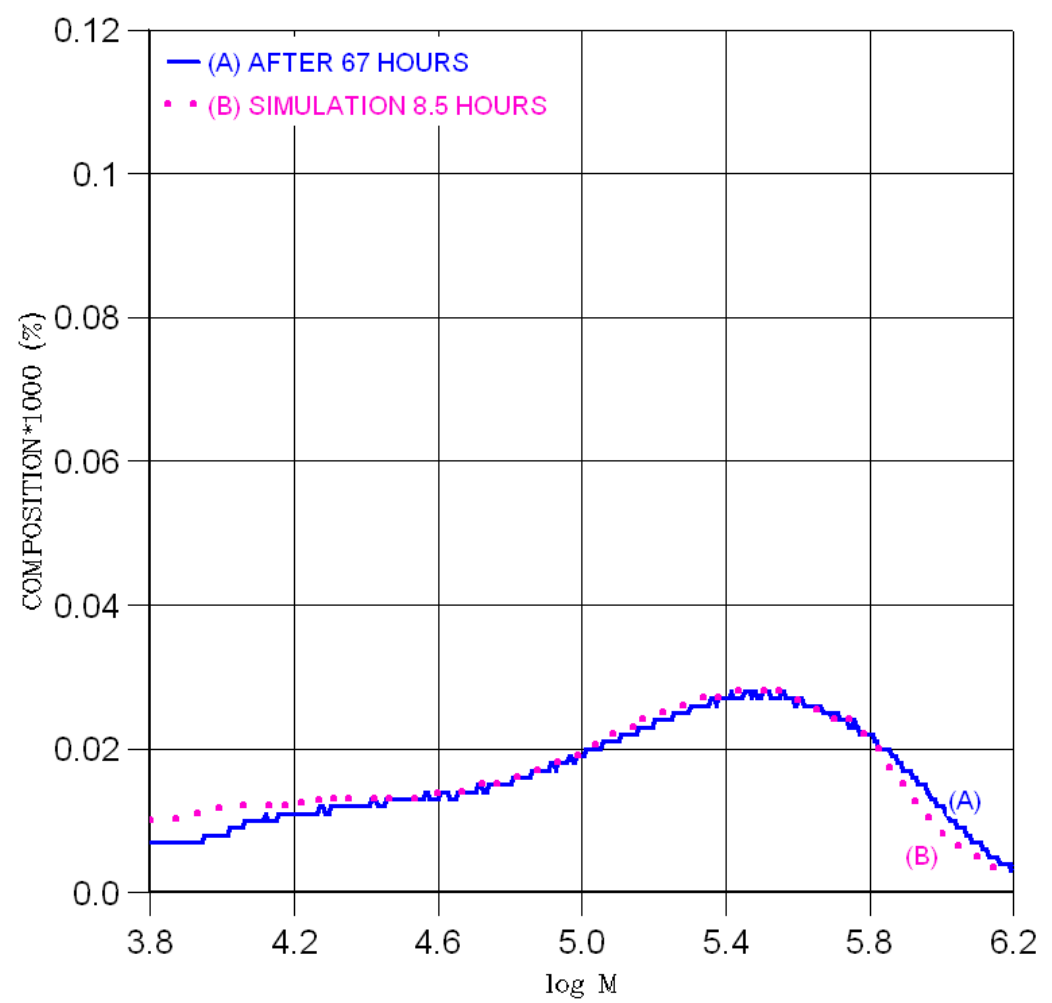

FigURE 5: Weight distribution after sixty-seven hours of incubation and simulation for 8.5 hours. 


$$
\mathcal{T}_{2}=\int_{0}^{T_{2}} \sigma(s) d s .
$$

Here we set $T_{1}=\mathcal{T}_{1}=5 / 24$ day according to the results shown in Figures 2 and 4 , and set $T_{2}=67 / 24$ day and $\mathcal{T}_{2}=8.5 / 24$ day in view of Figure 5 . Equations (10) and (12) lead to $\mathcal{T}_{1}=\left(e^{b} / a\right)\left(1-e^{-a T_{1}}\right)$. It follows from (10) that $\sigma(t)=a \mathcal{T}_{1} e^{-a t} /\left(1-e^{-a T_{1}}\right)$. It also follows from equation (11) that

$$
\tau=\mathcal{T}_{1} \frac{1-e^{-a t}}{1-e^{-a T_{1}}}
$$

Now equation (13) leads to

$$
\mathcal{T}_{2}=\mathcal{T}_{1} \frac{1-e^{-a T_{2}}}{1-e^{-a T_{1}}}
$$

Let

$$
h(a)=\frac{1-e^{-a T_{2}}}{1-e^{-a T_{1}}}-\frac{\mathcal{T}_{2}}{\mathcal{T}_{1}},
$$

which implies that equation (15) is equivalent to

$$
h(a)=0 .
$$

We solved equation (16) numerically for the values of parameters: $T_{1}=5 / 24$, $T_{2}=67 / 24, \mathcal{T}_{1}=T_{1}, \mathcal{T}_{2}=8.5 / 24$, and found an approximate value of the solution $a \approx 4.259$.

Once we find the time factor $\sigma(t)$ and the molecular factor $\lambda(M)$, we simulate the transition of the weight distribution by solving the initial value problem (2)-(3). The application of the trapezoidal rule to the integral on the right-hand side of equation (2) leads to the following system:

$$
\frac{d w_{i}}{d t}=\sigma(t)\left(-\lambda_{i} w_{i}+\Delta M F_{i}\right), \quad i=0,1,2, \ldots, N,
$$

where $\Delta M=(b-a) / N, M_{i}=a+i \Delta M, \lambda_{i}=\lambda\left(M_{i}\right)$, and

$$
F_{i}=\frac{1}{2} \lambda_{i} w_{i}+\sum_{j=i+1}^{N-1} \lambda_{j} w_{j}+\frac{1}{2} \lambda_{N} w_{N} .
$$


The unknown variable $w_{i}=w_{i}(t)$ denotes an approximate value of $w\left(t, M_{i}\right)$. Here we chose the trapezoidal rule for its convenience. The system (17) is associated with the initial condition

$$
w_{i}(0)=f_{i}=f\left(M_{i}\right), \quad i=0,1,2, \ldots, N .
$$

We set the value of the parameter $N=4000$, and applied the AdamsBashforce-Moulton predictor and corrector in PECE mode in conjunction with Runge-Kutta method [5] with the steplength $1 / 4800$ to solve the initial value problem (17)-(18) numerically. Figure 6 shows the result of the numerical simulation for the weight distribution of PLA for sixty-seven hours of incubation.

\section{Discussion}

During the incubation, PLA was dissolved in chloroform. The decrease in degradation rate was due to evaporation of chloroform. The amount of chloroform decreased exponentially, and we assumed that the degradation rate was given by the expressions (5) and (10). The degradation rate shown in Figure 2 was based only on the weight distribution before and after incubation for five hours. Nevertheless Figure 6 shows an acceptable correspondence between the experimental result and the numerical result for the weight distribution after incubation for sixty-seven hours, which justifies the model with temporally dependent degradation rate (2), (5) and (10).

The time independent model analyzed previously,

$$
\frac{\partial w}{\partial t}=-\lambda(M) w+\int_{M}^{\infty} \frac{2 M}{K^{2}} \lambda(M) w(t, K) d K,
$$

was derived under the assumption that the number of degraded molecules of molecular weight $M$ should be uniformly distributed over the weight interval $[0, M][12,13,17,18]$. Here we derive the endogenous depolymerization 


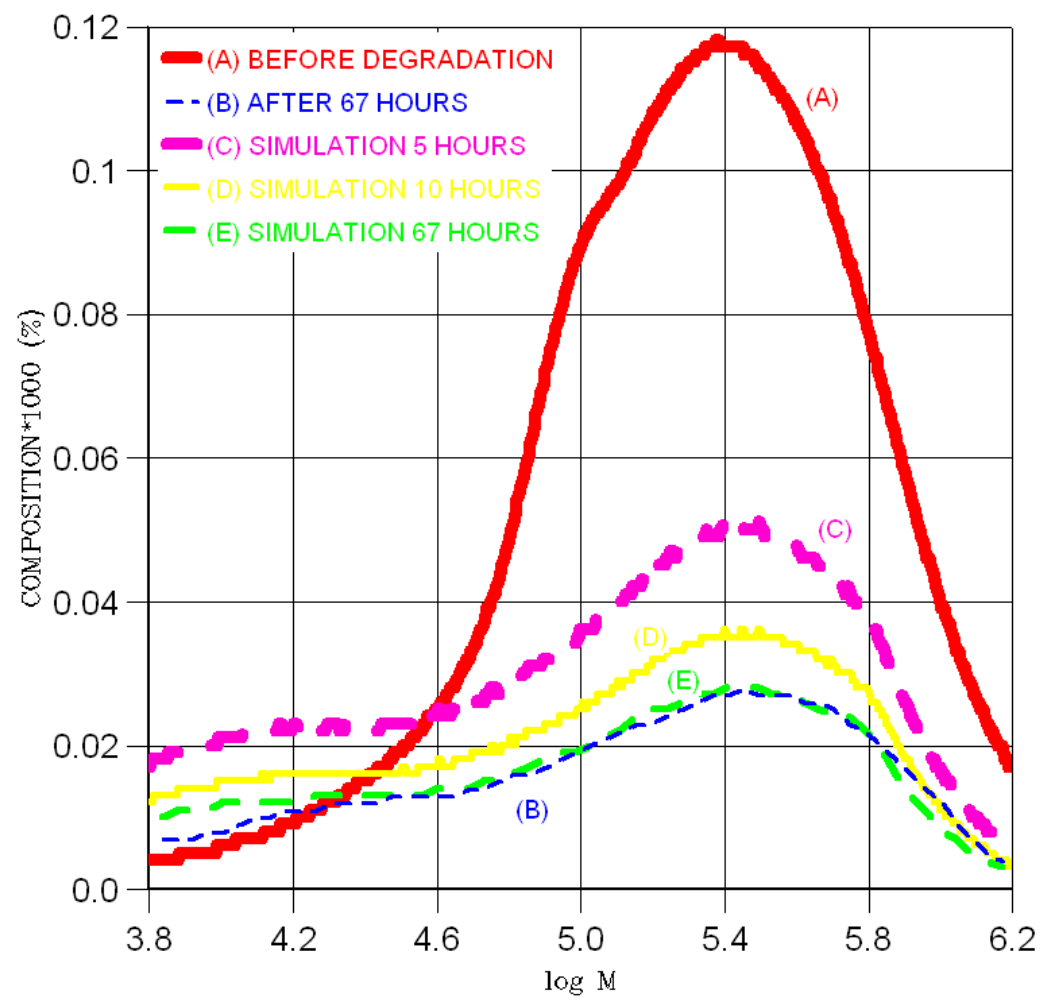

Figure 6: The weight distribution of PLA for sixty-seven hours of incubation. 
model (2) under the assumption that the total weight of degraded molecules of molecular weight $M$ should be uniformly distributed over the interval, and obtained the corresponding time independent model (6) from equation (2). The degradation rate of the time independent model (6) shown in Figure 2 is smaller than the degradation rate of the model (19). Nevertheless Figures 3 and 4 justify the time independent model (6).

The degradation rate shown in Figure 2 is an average degradation rate over five hours. Figure 5 shows that it would only take 8.5 hours with the average degradation rate to reach the stage after actual incubation for sixtyseven hours, which shows a loss of degradability after the first five hours of incubation. Figure 6 shows that molecules broke down rapidly at an early stage of incubation, and the degradation slowed down quickly as chloroform was lost by evaporation.

The mathematical models similar to the endogenous depolymerization model (2) or (6) have been introduced by other authors. Those include the governing equation for particle-size distribution in simultaneous binary fragmentation and aggregation reactions or the population balance equation $[6,8,10]$, the equation for mass balance for the polymer subject to random chain scission, repolymerization reactions, and chain-end scission [9], and the integro-partial differential equation of a first-order bond-breaking process for random scission and a first-order recombination process [11].

Acknowledgement This work is supported in part by a JSPS (MEXT) Grant-in-Aid for Scientific Research (C) (16540106).

\section{References}

[1] F. Kawai, Sphingomonads involved in the biodegradation of xenobiotic polymers, Journal of Industrial Microbiology 83 Biotechnology 23 
(1999), 400-407. C458

[2] F. Kawai, M. Shibata, S. Yokoyama, S. Maeda, K. Tada, and S. Hayashi, Biodegradability of Scott-Gelead photodegradable polyethylene and polyethylene wax by microorganisms, Macromolecular Symposia 144 (1999), 73-84. C458

[3] F. Kawai, M. Watanabe, M. Shibata, S. Yokoyama, and Y. Sudate, Experimental analysis and numerical simulation for biodegradability of polyethylene, Polymer Degradation and Stability 76 (2002), 129-135. doi:10.1016/S0141-3910(02)00006-X C458

[4] F. Kawai, M. Watanabe, M. Shibata, S. Yokoyama, Y. Sudate, and S. Hayashi, Comparative Study on Biodegradability of Polyethylene Wax by Bacteria and Fungi, Polymer Degradation and Stability 86 (2004), 105-114. doi:10.1016/j.polymdegradstab.2004.03.015 C458

[5] J. D. Lambert, Computational Methods in Ordinary Differential Equations (John Wiley and Sons, Chichester, 1973). C468

[6] G. Madras and B. J. McCoy, Numerical and similarity solutions for reversible population balance equations with size-dependent rates, Journal of Colloid and Interface Science 246 (2002) 356-365. doi:10.1006/jcis.2001.8073 C470

[7] S. Matsumura, N. Tomizawa, A. Toki, K. Nishikawa and K. Toshima, Novel Poly(vinyl alcohol)-degrading enzyme and the degradation mechanism, Macromolecules 32 (1999), 7753-7761. C459

[8] B. J. McCoy and G. Madras, Evolution to similarity solutions for fragmentation and aggregation, Journal of Colloid and Interface Science 201 (1998) 200-209. doi:10.1006/jcis.1998.5434 C470

[9] B. J. McCoy, Distribution Kinetics for Temperature-Programmed Pyrolysis, Industrial \& Engineering Chemistry Research 38 (1999) 4531-4537. C470 
[10] B. J. McCoy and G. Madras, Discrete and continuous models for polymerization and depolymerization, Chemical Engineering Science 56 (2001) 2831-2836. doi:10.1016/S0009-2509(00)00516-9 C470

[11] J. E. J. Staggs, A continuous model for vaporization of linear polymers by random scission and recombination, Fire Safety Journal 40 (2005) 610-627. doi:10.1016/j.firesaf.2005.05.004 C470

[12] M. Watanabe and F. Kawai, Numerical simulation for enzymatic degradation of poly (vinyl alcohol), Polymer Degradation and Stability 81 (2003), 393-399. doi:10.1016/S0141-3910(03)00122-8 C459, C468

[13] M. Watanabe and F. Kawai, Analysis of polymeric biodegradability based on experimental results and numerical simulation, Environmental Research and Control (in Japanese) 25 (2003), 25-32. C458, C459, C468

[14] M. Watanabe, F. Kawai, M. Shibata, S. Yokoyama, and Y. Sudate, Computational method for analysis of polyethylene biodegradation, Journal of Computational and Applied Mathematics 161 (2003), 133-144. doi:10.1016/S0377-0427(03)00551-X C458

[15] M. Watanabe, F. Kawai, M. Shibata, S. Yokoyama, Y. Sudate, and S. Hayashi, Analytical and computational techniques for exogenous depolymerization of xenobiotic polymers, Mathematical Biosciences 192 (2004) 19-37. doi:10.1016/j.mbs.2004.06.006 C458

[16] M. Watanabe and F. Kawai, Numerical simulation of microbial depolymerization process of exogenous type, R. May and A. J. Roberts (Eds.) Proc. of 12th Computational Techniques and Applications Conference, CTAC-2004, ANZIAM Journal, 46(E), pp. C1188-C1204, 2005. http://anziamj . austms .org. au/V46/CTAC2004/Wata C458

[17] M. Watanabe and F. Kawai, Mathematical modelling and computational analysis of enzymatic degradation of xenobiotic 
polymers, Applied Mathematical Modelling 30 (2006) 1497-1514. C459, C462, C468

[18] M. Watanabe, F. Kawai, S. Tsuboi, S Nakatsu, and H. Ohara, Study on enzymatic hydrolysis of polylactic acid by endogenous depolymerizaion model, Macromolecular Theory and Simulations 16 (2007) 619-626. doi:10.1002/mats.200700015 C459, C460, C462, C468

[19] M. Watanabe and F. Kawai, Mathematical study of the biodegradation of xenobiotic polymers with experimental data introduced into analysis, Proceedings of the 7th Biennial Engineering Mathematics and Applications Conference, EMAC-2005, Melbourne, Editors: Andrew Stacey and Bill Blyth and John Shepherd and A. J. Roberts, ANZIAM J. 47 pp. C665-C681, 2007.

http://anziamj.austms.org.au/V47EMAC2005/Watanabe C458 


\section{Author addresses}

1. M. Watanabe, Graduate School of Environmental Science, Okayama University, 1-1, Naka 3-chome, Tsushima, Okayama 700-8530, JAPAN. mailto: watanabe@ems. okayama-u.ac.jp

2. F. Kawai, Research Institute for Bioresources, Okayama University. 